\title{
Central $\alpha$-1-Adrenergic Control of Vasopressin Secretion in Newborn Lambs
}

\author{
STEVEN M. BLOCK, KAREN D. BARNES, AND REBECCA ZOLTOSKI \\ Perinatal Research Laboratory, Departments of Pediatrics and Physiology/Pharmacology, The Bowman Gray \\ School of Medicine of Wake Forest University, Winston-Salem, North Carolina 27103
}

\begin{abstract}
The hypothesis that central $\alpha-1$ adrenoceptors are inhibitory to the hypotension-induced secretion of vasopressin was tested by subjecting lambs that were instrumented for a long term to varying degrees of hypotension after intracerebroventricular injections of prazosin or placebo. Eight lambs in the 1st wh of life treated with intracerebroventricular injections of placebo had their mean arterial blood pressures decreased 14 and $21 \%$ by i.v. infusion of nitroprusside. Arginine vasopressin levels rose to $7.3 \pm 2.4 \mathrm{pmol} / \mathrm{L}$ only with the greater degree of hypotension. When the lambs were treated with intracerebroventricular injections of $1 \mu \mathrm{g} / \mathrm{kg}$ of prazosin, the blood pressures were decreased 13 and $23 \%$, and the vasopressin levels were $15.4 \pm 16.6$ and $27.5 \pm 20.3 \mathrm{pmol} / \mathrm{L}$, respectively. A relationship was shown between the degree of hypotension and the plasma arginine vasopressin levels with both the placebo and prazosin, the slope being much steeper for the prazosin treatment $(-1.11)$ than for the placebo treatment $(\mathbf{- 0 . 3 1})$. Plasma renin activity was increased a similar amount in both groups, and there was no change in plasma cortisol levels. We conclude that $\alpha-1$ adrenoceptors in the brain are inhibitory to the secretion of arginine vasopressin. These results differ from observations in adult rats and dogs and may be accounted for by developmental or species differences. (Pediatr Res 30: 5054,1991 )
\end{abstract}

\section{Abbreviations}

AVP, arginine vasopressin

CSF, cerebrospinal fluid

HR, heart rate

$\mathrm{ICV}$, intracerebroventricular

MABP, mean arterial blood pressure

PE, phenylephrine

PRA, plasma renin activity

AVP has been shown to have a role in fetal and neonatal cardiovascular homeostasis as a "stress" hormone (1-3). Neonatal and fetal AVP secretion may be induced by hypoxia, hypotension, and acidosis. Levels of this hormone are particularly high around the time of delivery, especially in the case of fetal asphyxia (3).

Although there is an increasing body of knowledge concerning the control of AVP secretion in adult animals, little is known about the neonate. CNS $\alpha-1$ and $\alpha-2$ adrenoceptors are involved

Received November 6, 1990; accepted March 11, 1991.

Correspondence and reprint requests: Steven M. Block, M.B., B.Ch., Department of Pediatrics, The Bowman Gray School Of Medicine, 300 South Hawthorne Road, Winston-Salem NC 27103

Supported by Grant HD 23847 and a grant from the Brenner Children's in the regulation of AVP secretion in adult animals (4-6). We have previously reported that lamb fetuses treated with i.v. prazosin, an $\alpha-1$ adrenoceptor blocker, and subsequently subjected to hypotensive hemorrhage had excessive levels of AVP when compared with similar hemorrhagic hypotension without prazosin (7). This observation led us to hypothesize that hypothalamic $\alpha-1$ receptors exert an inhibitory influence on the secretion of AVP in response to hypotension in the near-term lamb fetus. In the neonatal lamb, AVP levels after hemorrhage were similar with prazosin and with vehicle, but the relative decrease in blood pressure was less in the prazosin-treated lambs and may have been subthreshold for the secretion of AVP (2). To prove our hypothesis, we designed an experiment in which CNS $\alpha$-1 adrenoceptor blockade was achieved in the newborn lamb by ICV injection, rather than i.v. injection of prazosin. In so doing, we hoped to avoid the potential confounding systemic effects of systemic blockade.

\section{MATERIALS AND METHODS}

Animal preparation. All procedures were approved by the Animal Care and Use Committee of the Bowman Gray School of Medicine of Wake Forest University.

Twelve lambs were operated on within $48 \mathrm{~h}$ of birth under ketamine anesthesia $(20 \mathrm{mg} / \mathrm{kg})$. In addition, $1 \%$ lidocaine was used locally at all incision sites. The animals were carefully shaved, and all procedures were done with strict sterile technique.

With the animal supine, incisions were made over both femoral arteries. The femoral arteries and veins were identified, isolated, and cannulated with polyvinyl catheters. The catheter tips were advanced to the level of the bifurcation of the aorta. The catheters were tunneled s.c. to the animal's flank and stored in a canvas pouch. The skin incisions were sutured closed.

The animal was then placed in the prone position. A midline scalp incision was made, and the scalp reflected laterally. A small burr hole was made in the skull $0.5 \mathrm{~cm}$ posteriorly and lateral to the bregma. A 20-gauge Teflon catheter (Angiocath; Deseret Medical Inc., Sandy, UT) was shortened to $1.5-\mathrm{cm}$ length and positioned on its trochar so that the trochar barely protruded from the catheter tip. It was inserted through the burr hole into the lateral ventricle of the brain, and its position was confirmed by the presence of CSF within the catheter. The trochar was then removed from the catheter, and a very fine polyvinyl catheter (V1; Bolab, Lake Havasu City, AZ) was inserted so that its tip was at the end of the Teflon catheter $1.5 \mathrm{~cm}$ beneath the skull surface. The polyvinyl catheter was held in place, and the Teflon catheter withdrawn over it. The polyvinyl catheter was sutured to the periosteum of the skull, using a 6-0 nylon suture. A drop of cyanoacrylate cement was applied to the burr hole, the catheter, and the suture. The catheter was tunneled posteriorly to the nape of the neck, and the scalp incision sutured. The catheter was secured in a "stockinet" cap fashioned individually for each
lamb.

Upon recovery from surgery (usually within $1 \mathrm{~h}$ ), the lambs 
were returned to their mothers' pens. They were allowed to recover for at least $48 \mathrm{~h}$ before the 1 st study. The lambs were only studied after they had demonstrated consistent weight gain for at least $2 \mathrm{~d}$.

Dose-response experiment. Four animals were studied to establish an ICV prazosin dose for use in the hypotension experiment.

The animals were allowed to feed and then taken from the ewes and placed in a modified Pavlov sling where they rested comfortably with their feet touching the floor beneath them. The arterial lines were attached to pressure transducers (Statham P23dB; Gould Electronics, Cleveland, $\mathrm{OH}$ ) and a blood pressure and HR recorder (Gould Electronics). The animals were allowed to stabilize for at least $30 \mathrm{~min}$ before any manipulations. Each lamb was studied on 3 separate days; a different dose of prazosin was administered on each day. The order of dosing was randomized. Doses used were $0.25,0.5$, and $1.0 \mu \mathrm{g} / \mathrm{kg}$.

Prazosin was solubilized in DMSO and made up to volume with artificial sheep CSF (8), warmed to $39^{\circ} \mathrm{C}$, and corrected to a pH of 7.40 by bubbling $\mathrm{CO}_{2}$ gas through it. The volume injected with each dose was $0.05 \mathrm{~mL}$.

After a dose of prazosin was injected as a bolus into the ICV catheter, the animal's HR and blood pressure were recorded for $1 \mathrm{~h}$. At that point, an i.v. dose of PE [24.5 nmol $/ \mathrm{kg}(5 \mu / \mathrm{kg})]$, an $\alpha-1$ adrenergic agonist, was given, and the $\mathrm{HR}$ and blood pressure were observed. The dose of PE was chosen because it produces a small (approximately 20\%) change in blood pressure. Any diminution of the blood pressure increase would suggest a systemic effect of the prazosin. After all three doses were given, the animal was killed with an overdose of pentobarbital, and saturated potassium chloride was given i.v. Methylene blue was injected into the ICV catheter, the brain dissected, and the location of the catheter confirmed by the presence of methylene blue within the lateral cerebral ventricle.

Hypotension experiment. We employed a randomized crossover design. Each lamb was studied four times. On two occasions prazosin and on two occasions vehicle were injected into the ICV catheter. As above, the prazosin was dissolved in DMSO and diluted up to volume in artificial sheep CSF controlled for temperature and $\mathrm{pH}$. The volume injected was $0.05 \mathrm{~mL}$ in all cases. For control experiments, a proportional mixture of DMSO and artificial sheep CSF without prazosin was used.

Each experiment began with a 30 -min stabilization period after which an i.v. dose of PE $(24.5 \mathrm{nmol} / \mathrm{kg})$ was given to observe the magnitude of the cardiovascular response for comparison with the response at the end of the experiment. Once blood pressure and HR had returned to normal, the animal was allowed to rest for an additional $15 \mathrm{~min}$ before any manipulations. Two control blood samples were drawn 10 min apart. The dose of prazosin or an equal volume of placebo was given as a bolus via the ICV catheter. Nitroprusside was then infused i.v. with a variable rate infusion pump. The administration rate of nitroprusside was adjusted to produce either mild (10-15\%) or moderate (20-25\%) hypotension. Blood samples were drawn 5 and $10 \mathrm{~min}$ after the desired level of hypotension was achieved. The nitroprusside infusion was then discontinued, and the animal observed until the blood pressure returned to control levels and stabilized. A second dose of PE $(24.5 \mathrm{nmol} / \mathrm{kg})$ was given i.v. and the effect on HR and blood pressure recorded.

The order of study was randomized for each lamb. Each experiment was performed on a separate day. Between experiments, the lambs were returned to their mothers. They remained well and continued to gain weight.

On completion of all the experiments, the lambs were killed, and the position of the ICV catheters confirmed by the methylene blue technique described above.

Blood samples were analyzed for $\mathrm{pH}, \mathrm{PCO}_{2}, \mathrm{PO}_{2}, \mathrm{AVP}, \mathrm{PRA}$, cortisol, and osmolality.

Arterial blood gases and $\mathrm{pH}$ were measured using a Radiometer ABL MK 3 blood gas analyzer (Radiometer, Copenhagen, Denmark). Osmolality was determined using the freezing point depression method (Advanced Instruments Needham Heights, MA). AVP was measured using a RIA, as described previously (9). Interassay coefficient of variability for the assay was less than $11 \%$, and intraassay coefficient of variability was less than $8 \%$. PRA was measured using a commercially available kit (Biotecx, Friendswood, Texas), with inter- and intraassay coefficients of variability of less than 10 and $8 \%$, respectively. Cortisol determinations were made using a RIA (9). The inter- and intraassay coefficients of variability were less than 12 and $8 \%$, respectively.

Statistical analysis. All results are reported as mean \pm SEM, except where indicated. Two-factor analysis of variance, corrected for repeated measures on time, was used to evaluate changes in all measured continuous variables with respect to time and group effects. Post-hoc comparisons were made using the Dunnett and Newman/Keuls tests. Regression analysis was performed where indicated, using the mean least-squares method. The Mann-Whitney test was used to compare group slopes where the variances were nonhomogeneous.

\section{RESULTS}

Dose-response experiment. Four animals were studied to establish an ICV dose of prazosin that would likely produce CNS $\alpha-1$ blockade but would not change the resting blood pressure.

Baseline blood pressures were $79 \pm 3.2,78 \pm 3.3$, and $72 \pm$ $2.8 \mathrm{~mm} \mathrm{Hg}$, respectively, for the experiments where $0.25,0.5$, and $1.0 \mu \mathrm{g} / \mathrm{kg}$ of prazosin were injected ICV. These blood pressures did not change over the hour of observation after any of the doses. A $24.5-\mathrm{nmol} / \mathrm{kg}$ dose of $\mathrm{PE}$ given i.v. yielded increases in MABP of $27 \pm 6.2(26 \pm 3.1 \%), 15 \pm 1.1(19 \pm$ $1.8 \%)$, and $17 \pm 7.0 \mathrm{~mm} \mathrm{Hg}(22 \pm 4.6 \%)$ after the three prazosin doses, respectively. The blood pressure increases were significant $(p<0.05)$, whereas the interdose differences were not. Mean resting HR were $179 \pm 13,201 \pm 14$, and $235 \pm 18 \mathrm{bpm}$ in the three groups, respectively. HR fell in response to PE $30.6 \pm 3.6$, $24 \pm 3.3$, and $28 \pm 2.1 \%$, respectively. Again, the response to PE was significant, although there was no difference between the three doses. On the basis of these results, a prazosin dose of 1 $\mu \mathrm{g} / \mathrm{kg}$ was selected for the hypotension study.

Hypotension study. Eight lambs were studied in a crossover fashion under the following conditions: Control with 10 to $15 \%$ hypotension (C-mild); control with 20 to $25 \%$ hypotension (Cmoderate); prazosin with 10 to $15 \%$ hypotension (P-mild); and prazosin with 20 to $25 \%$ hypotension (P-moderate).

Cardiovascular measurements. Baseline cardiovascular measurements are shown in Table 1 . The baseline values were similar in all groups. In the C-mild group, before the ICV injection of artificial CSF, the MABP increased $26 \pm 3 \%$. At the end of the experiment, the MABP rose $25 \pm 2 \%$. In the other groups, the MABP increases were as follow: C-moderate, $19 \pm 2$ and $21 \pm$ $2 \%$, P-mild, $26 \pm 5$ and $27 \pm 4 \%$; and P-moderate, $29 \pm 4$ and $25 \pm 7 \%$ before the ICV dose and at the end of the experiment, respectively. There were no differences between the pretreatment and posttreatment values in any group, confirming the absence of arterial $\alpha-1$ adrenoceptor blockade.

MABP and HR changes in response to nitroprusside are seen in Figure 1. All MABP values were different from baseline. Pmild and C-mild values did not differ from one another. Similarly, P-moderate and C-moderate values did not differ. Differences between the mild and moderate degrees of hypotension were significant in both prazosin and control groups $(p<0.01)$ HR was similar among the groups at baseline and increased significantly with hypotension in all animals $(p<0.01)$. The HR increase was greater in the $\mathrm{C}$-mild than in the $\mathrm{C}$-moderate experiments $(p<0.01)$ but not statistically different from the prazosin experiments.

Blood gases and osmolality. Baseline blood gases and osmolality are shown in Table 1. There were no significant differences among the groups, and they did not change during the experiments. 
Table 1. Baseline cardiovascular, blood gas, and osmolality values*

\begin{tabular}{lcccccc}
\hline \multicolumn{1}{c}{ Study } & $\begin{array}{c}\mathrm{MABP} \\
(\mathrm{mm} \mathrm{Hg})\end{array}$ & $\begin{array}{c}\mathrm{HR} \\
(\mathrm{bpm})\end{array}$ & $\mathrm{pH}$ & $\begin{array}{c}\mathrm{PaCO}_{2} \\
(\mathrm{kPa})\end{array}$ & $\begin{array}{c}\mathrm{PaO}_{2} \\
(\mathrm{kPa})\end{array}$ & $\begin{array}{c}\mathrm{Osmolality} \\
(\mathrm{mmol} / \mathrm{kg})\end{array}$ \\
\hline C-mild & $70.5 \pm 1.5$ & $231 \pm 20$ & $7.38 \pm 0.01$ & $4.87 \pm 0.19$ & $11.33 \pm 0.57$ & $286 \pm 3$ \\
C-moderate & $68.7 \pm 1.3$ & $239 \pm 9$ & $7.33 \pm 0.02$ & $5.25 \pm 0.27$ & $12.21 \pm 0.83$ & $275 \pm 4$ \\
P-mild & $68.1 \pm 2.1$ & $207 \pm 16$ & $7.39 \pm 0.07$ & $4.85 \pm 0.24$ & $11.56 \pm 0.43$ & $281 \pm 4$ \\
P-moderate & $68.5 \pm 2.5$ & $234 \pm 17$ & $7.40 \pm 0.02$ & $5.13 \pm 0.27$ & $11.01 \pm 0.39$ & $288 \pm 4$ \\
\hline
\end{tabular}

* All values are expressed as mean \pm SEM. $\mathrm{C}$ and $\mathrm{P}$ refer to the control and prazosin experiments, respectively. Mild and moderate refer to the degree of hypotension, about 14 and $23 \%$, respectively. $\mathrm{PaCO}_{2}$, arterial $\mathrm{CO}_{2}$ tension; $\mathrm{PaO}_{2}$, arterial $\mathrm{O}_{2}$ tension.

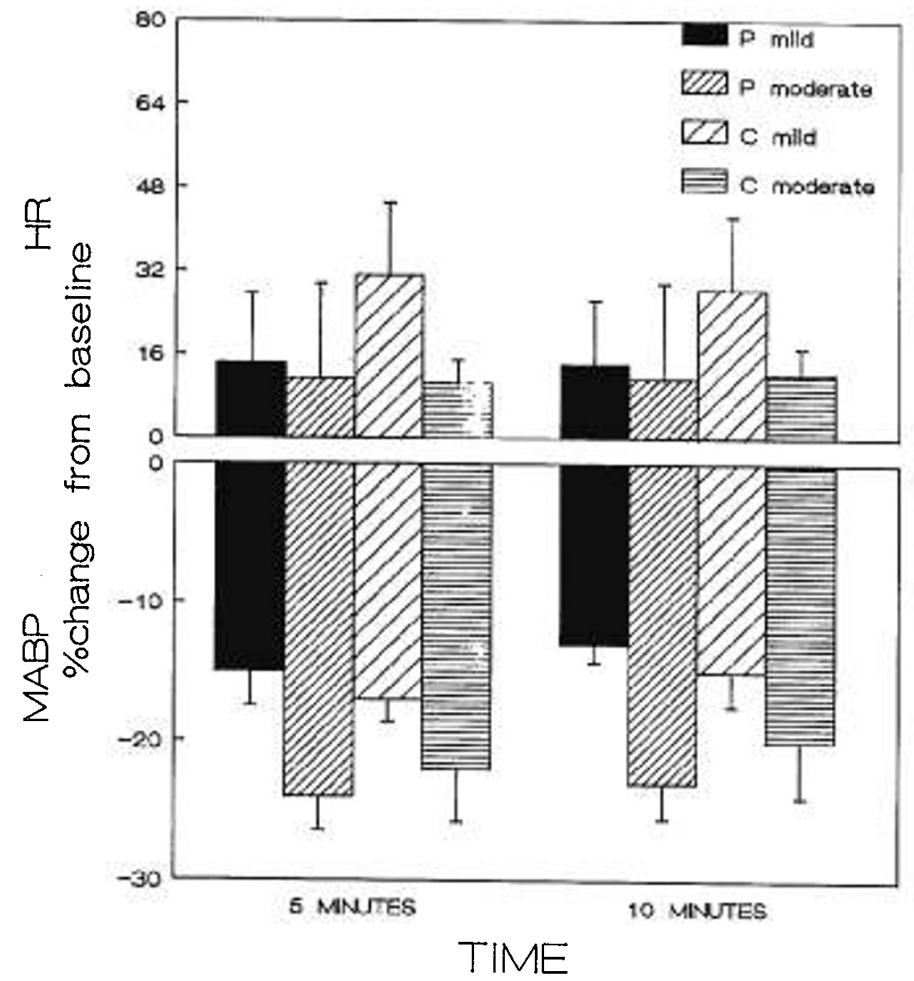

Fig. 1. Blood pressure and HR. Changes from baseline in HR (upper panel) and MABP (lower panel) 5 and $10 \mathrm{~min}$ after the onset of nitroprusside infusion. Blood pressures differed significantly $(p<0.01)$ between the mild and moderate groups but not between the treatments at similar levels of hypotension.

Vasopressin. Baseline AVP levels were $1.4 \pm 0.5$ and $1.3 \pm 0.4$ $\mathrm{pmol} / \mathrm{L}$ in the $\mathrm{C}$-mild and $\mathrm{C}$-moderate experiments and $2.3 \pm$ 0.7 and $1.6 \pm 0.4 \mathrm{pmol} / \mathrm{L}$ in the P-mild and P-moderate experiments, respectively. There were no significant differences among the groups. Changes in AVP levels are shown in Figure 2. AVP levels in the prazosin treatment experiments were higher at both 5 and 10 min of hypotension than in the animals treated with the vehicle $(p<0.01)$. There was no statistical difference between the AVP levels when comparing the P-mild and P-moderate experiments. More modest increases in AVP were seen in the control experiments; the increases were significant only for the C-moderate experiments.

There appeared to be a tendency for increased AVP levels with the greater degree of hypotension. The level of hypotension achieved with each experiment and at each time point varied somewhat, allowing us to plot AVP levels against the change in blood pressure for all of the animals together (Fig. 3). A significant relationship exists between the degree of hypotension and the AVP level, with the slope of the curve being much steeper for the prazosin experiments $(\mathrm{y}=-1.11 \mathrm{x}+0.68, r=-0.6431$, $p<0.00001)$ than the control experiments $(\mathrm{y}=-0.31 \mathrm{x}+1.18$, $r=-0.446, p<0.0001)$.

The prazosin and control experiments for each lamb were pooled so that a regression line over a range of blood pressures could be drawn for each individual subject under each of the

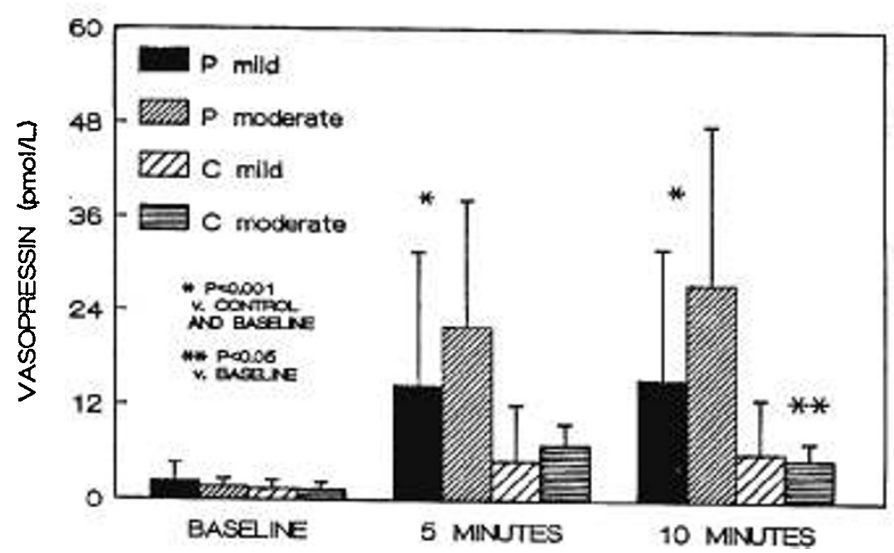

Fig. 2. Vasopressin. AVP levels at baseline and 5 and $10 \mathrm{~min}$ after the onset of hypotension. Significant differences exist between the prazosin and control experiments at both levels of hypotension $(p<0.001)$. AVP levels differed significantly from baseline $(p<0.05)$ in the control experiments, with moderate hypotension only at $10 \mathrm{~min}$.

drug treatments. All of the prazosin experiments were then treated as one group and the control experiments as another. The median slope for the prazosin experiments was -0.78 (variance 1.220) and for the control experiments -0.095 (variance $0.096)$. The groups were statistically different $(p<0.05$, MannWhitney).

Plasma renin activity. PRA results are shown in Table 2 . There was an increase in PRA with hypotension in both mild and moderate hypotension categories. PRA levels were higher with greater degrees of hypotension, although statistical significance was not reached. Because of this apparent trend, we submitted the data to regression analysis and showed that PRA increased linearly as the blood pressure fell $(p<0.0001)$. The regression equations were $\mathrm{y}=-0.32 \mathrm{x}+6.5(r=-0.41, p<0.0095)$ and $\mathrm{y}$ $=-0.49 \mathrm{x}+7.4(r=-0.582, p<0.0001)$ in the control and prazosin experiments respectively. The pooled mean slopes were not significantly different.

Cortisol. Cortisol results are shown in Table 2. There were no changes in cortisol levels with either mild or moderate hypotension, with or without prazosin.

\section{DISCUSSION}

This study provides important information concerning the control of AVP secretion in the newborn lamb. We have shown that lambs, instrumented on a long-term basis, treated with prazosin ICV and then made hypotensive have higher circulating levels of AVP than they do when they are made hypotensive under control conditions. AVP levels are directly related to the decrease in blood pressure. Alpha-1 adrenergic blockade exaggerates this relationship, greatly increasing AVP levels for similar degrees of hypotension. The increased AVP levels are not associated with changes in $\mathrm{pH}$ or osmolality, and other hormones, which are secreted during hypotension, are unaffected by $\alpha-1$ blockade. The results cannot be accounted for by peripheral $\alpha-1$ adrenoceptor blockade, because the hypertensive response to i.v. PE was unaffected. We conclude that CNS $\alpha-1$ adrenoceptors 


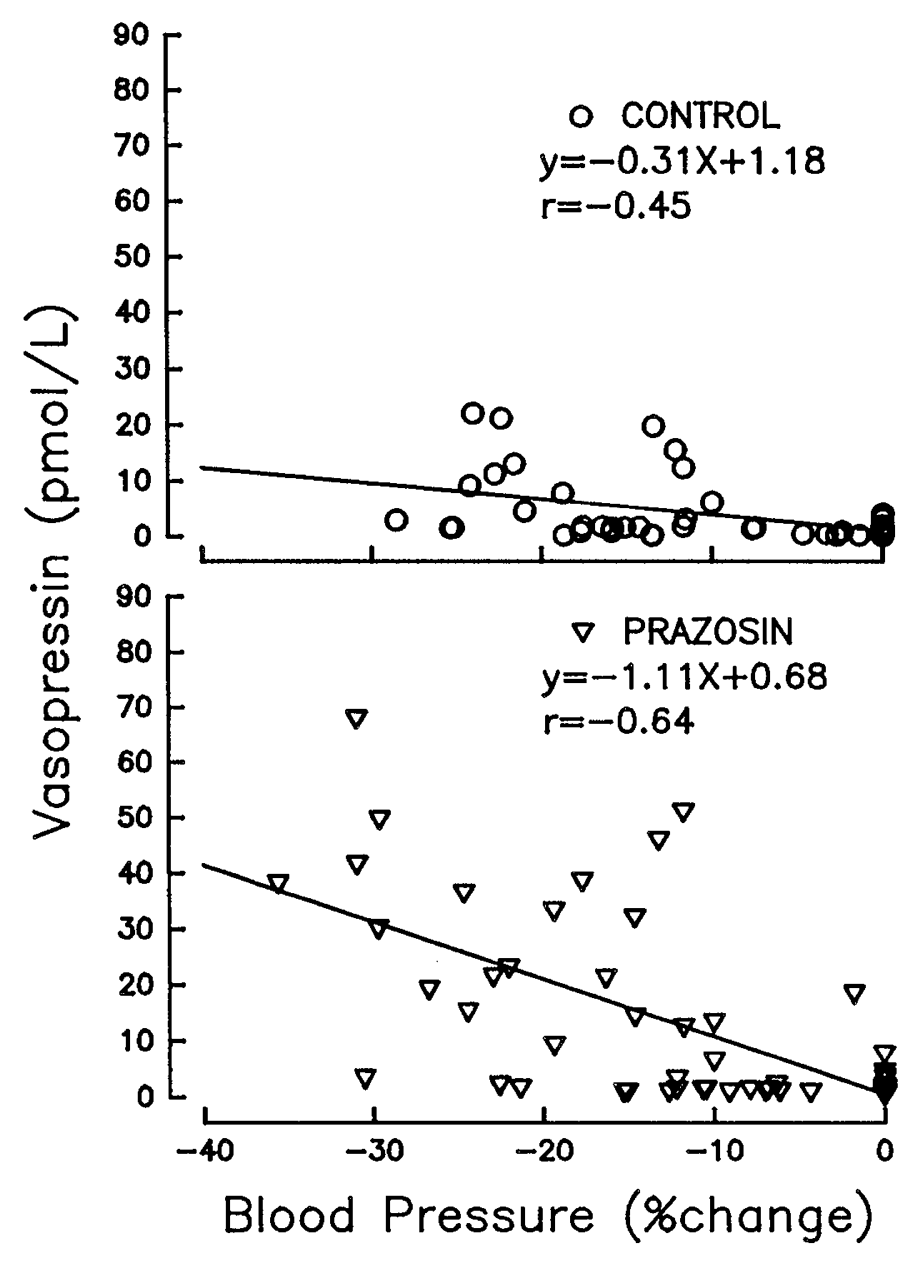

Fig. 3. Vasopressin. Plasma vasopressin levels plotted against the percentage of decrease in MABP. The upper panel represents the contro experiments, and the lower panel represents the prazosin experiments.

are inhibitory to the hypotension-induced secretion of AVP in the conscious lamb.

We based our hypothesis that central $\alpha$ - 1 adrenoceptors inhibit hypotension-induced AVP secretion on our previous report of exaggerated AVP secretion with hemorrhagic hypotension after i.v. injections of prazosin in fetal lambs (7). We did not observe a similar effect in the neonatal lambs treated with i.v. prazosin (2) where the change in blood pressure induced by hemorrhage was only $14 \%$ in the prazosin-treated lambs and was probably too small to result in large increases in AVP levels.

We speculate that the differences seen between the results shown here and reported elsewhere are ontogenetically based, although we cannot rule out interspecies differences and differences based on experimental design. Developmental differences have been shown in several aspects of the physiology of vasopressin. There is a clear developmental influence on its secretion in response to hypotension during fetal life in the sheep (1). Also,
AVP is present very early in fetal life $(10,11)$ and appears important in the stimulation of ACTH secretion. Its relationship to the appearance of corticotrophin-releasing factor and its function in the stimulation of ACTH secretion change with development in both the sheep (10) and the rat (11). Thus, we speculate that the inhibitory effects reported here are developmentally based.

We are hampered by the absence of comparable literature regarding the adult sheep and therefore cannot comment fully on the ontogeny of the control of AVP secretion in this species. In our previously reported experiments (2), we showed that i.v. injections of prazosin followed by hemorrhagic hypotension yielded higher levels of AVP than we found when ewes were bled a similar volume under control conditions. However, a greater degree of hypotension in the prazosin-treated ewes in that study may account for the higher AVP levels and may have no bearing on the central effects of prazosin in the adult. Additional studies in juvenile and adult sheep must be done to allow a definitive comment about the role of $\alpha-1$ adrenoceptors in the control of AVP secretion during development.

Numerous experiments in a variety of animal models have ascribed both stimulatory and inhibitory roles to the $\alpha-1$ and $\alpha$ 2 adrenoceptors located on the AVP secreting neurons of the hypothalamus $(3,5,6,12-14)$. It is possible that the differences between these studies and ours could be from the basis of interspecies variation as well as from a difference in the developmental stage of the subjects.

Differences in study design could account for some of the lack of agreement between our study and others. Other reports include experiments under both anesthetized $(5,12,14)$ and conscious (13) conditions. To eliminate stimuli for AVP secretion other than hypotension, our results were obtained using a long-term model for ICV injections of pharmacologic agents in neonatal animals. Our technique is easily performed, and our animals were all in a physiologically stable condition at the time of study. All the lambs were growing well and had normal activity when they were in the pens with the ewes. During the studies, the lambs rested comfortably in the slings in the standing position. The experiments were performed immediately after the lambs were fed, and they showed no evidence of agitation during the studies. The absence of surgical and anesthetic stress is important in the sheep, as is the absence of auditory/emotional stress (15). Whereas isolated anesthetic stress may not be important in the study of AVP secretion in the rat (16), most experiments investigating the control of AVP secretion, using acute experimental models, have involved both anesthetic and surgical stress, with unknown effects on the results. Our study design avoids any such potential interference.

Other experiments (5) used ICV drug doses sufficient to change the resting blood pressure and to consequently potentially affect the physiologic responses of the animals. We, therefore, performed dose-response experiments to choose a dose demonstrated to have no systemic effects. Our dose-response experiments were not designed to evaluate relationships between the dose of prazosin given ICV and the degree of increase in AVP levels produced by a fixed decrease in blood pressure.

Our data suggest that there may be a threshold for AVP

Table 2. Plasma renin activity and cortisol*

\begin{tabular}{|c|c|c|c|c|c|c|}
\hline & \multicolumn{3}{|c|}{ PRA [ng/(L.s)] } & \multicolumn{3}{|c|}{ Cortisol (nmol/L) } \\
\hline & Baseline & $5 \mathrm{~min} \dagger$ & $10 \min \dagger$ & Baseline & $5 \mathrm{~min}$ & $10 \mathrm{~min}$ \\
\hline C-mild & $1.64 \pm 0.36$ & $2.92 \pm 0.47$ & $3.00 \pm 0.61$ & $295 \pm 128$ & $218 \pm 28$ & $254 \pm 39$ \\
\hline C-moderate & $1.89 \pm 0.28$ & $4.36 \pm 0.94$ & $4.28 \pm 0.72$ & $309 \pm 135$ & $287 \pm 129$ & $295 \pm 127$ \\
\hline P-mild & $1.25 \pm 0.17$ & $3.44 \pm 0.47$ & $3.47 \pm 0.39$ & $276 \pm 41$ & $240 \pm 55$ & $270 \pm 58$ \\
\hline P-moderate & $2.11 \pm 0.53$ & $6.08 \pm 1.00$ & $6.22 \pm 0.97$ & $168 \pm 55$ & $174 \pm 44$ & $315 \pm 74$ \\
\hline
\end{tabular}

* All values expressed as mean \pm SEM. C and P refer to the control and prazosin experiments, respectively. Mild and moderate refer to the degree of hypotension, about 14 and $23 \%$, respectively. Five and $10 \mathrm{~min}$ refer to the duration of hypotension.

$\uparrow$ PRA was significantly higher than baseline and rose with decreasing MABP, $p<0.0001$. 
secretion in the lamb with a decrease in MABP between 15 and $20 \%$. When the lambs were treated with vehicle, mild hypotension did not produce an increase in the level of AVP, although moderate hypotension produced a small but significant increase. Our data do not indicate whether the threshold for AVP secretion is altered by central $\alpha-1$ adrenergic blockade.

These data in combination with other reports from our laboratory give some insights into changes in sensitivity to hypotension of the hypothalamus and neurohypophysis with development. Some idea of how the degree of hypotension required for AVP secretion changes with development can be gained by comparison of our results with experiments in fetal lambs at various gestational ages (1). In our vehicle-treated newborn lambs, AVP levels were $7.2 \pm 2.4 \mathrm{pmol} / \mathrm{L}$, with a $22 \%$ decrease in blood pressure. The peak levels of AVP produced by a $45 \%$ decrease in blood pressure in fetuses at $<0.68$ gestation were 7.2 $\pm 2.5 \mathrm{pmol} / \mathrm{L}$, virtually identical to the levels seen with much less hypotension in the newborns. This comparison indicates that the neonate is more sensitive to hypotension in terms of AVP secretion, barring an increased metabolic clearance rate for AVP in the fetus causing the relatively low AVP levels. The near-term fetus is more sensitive to hypotension than the midgestation fetus and is qualitatively similar to the newborn lamb.

In the present study, we detected no increase in cortisol associated with the ICV prazosin. In contrast, fetal lambs treated with i.v. prazosin and subjected to hypotensive hemorrhage had increased levels of cortisol that were detected even before the onset of the hemorrhage (7). It is likely that the pituitary portal levels of AVP were elevated in the fetus, resulting in the secretion of ACTH. Brieu et al. (10) concluded that AVP is more important in the stimulation of ACTH secretion in fetuses younger than $138 \mathrm{~d}$ gestation than at term, with corticotropin-releasing factor assuming a progressively more important role. The fetal cortisol response and its absence in the neonates reflects this ontogenetic phenomenon. It is unlikely that the observation in the newborn lambs can be accounted for by a dose of prazosin insufficient to affect ACTH secretion, because there was a clear effect on AVP secretion when hypotension was induced.

The control of AVP secretion is complex, depending on a variety of stimuli, each of which may depend on different hy- pothalamic mechanisms. In addition to the $\alpha-1$ adrenergic system, other receptors have been shown to be involved $(5,6)$. The rapid changes occurring in AVP-related mechanisms in the nearterm fetus raise important questions about the development of this system. Studies in older sheep at a variety of ages and observations of a variety of stimuli as well as receptors are needed to gain a complete understanding.

\section{REFERENCES}

1. Rose JC, Meis PJ, Morris M 1981 Ontogeny of endocrine (ACTH, vasopressin, cortisol) responses to hypotension in lamb fetuses. Am J Physiol 240: E656E661

2. Block SM, Rose KC, Ernest JM, Flowe K, South S, Zimmerman C 1987 Cardiovascular and endocrine response to hemorrhage after $\alpha_{1}$ blockade in lambs and ewes. Am J Physiol 252:R314-R319

3. Pohjavuori M, Fyhrquist F 1980 Hemodynamic significance of vasopressin in the newborn infant. J Pediatr 97:462-465

4. Brooks DP, Share L, Crofton JT 1986 Central adrenergic control of vasopressin release. Neuroendocrinology 42:416-420

5. Kimura T, Shoji M, litake K, Ota K, Matsui K, Yoshinaga K 1984 The role of central $\alpha_{1}$ - and $\alpha_{2}$-adrenoceptors in the regulation of vasopressin release

6. Reid IA, Nolan PL, Wolf JA, Keil LC 1979 Suppression of vasopressin secretion by clonidine: effect of $\alpha$-adrenoceptor antagonists. Endocrinology 104:14031406

7. Block SM, Rose JC, Ray D, Rawashdeh N, Barnes KD 1988 Endocrine responses of fetal lambs to hemorrhage after $\alpha_{1}$-adrenergic receptor blockade. Am J Obstet Gynecol 159:1256-1262

8. Beal AM, Bligh J 1977 Electrolyte concentrations in sheep cerebrospinal fluid. Res Vet Sci 22:382-383

9. Rose JC, Morris M, Meis PJ 1981 Hemorrhage in newborn lambs: effects on arterial blood pressure, ACTH, cortisol and vasopressin. Am J Physiol

10. Brieu V, Tonon M-C, Lutz-Bucher B, Durand P 1989 Corticotropin-releasing factor-like immunoreactivity and ACTH-releasing bioactivity in the hypothalamic tissue from fetal and neonatal sheep. Neuroendocrinology 49:164-168

11. Rundle SE, Funder JW 1988 Ontogeny of corticotropin-releasing factor and arginine vasopressin in the rat. Neuroendocrinology 47:374-378

12. Reid IA, Ahn JN, Trinh T, Shackelford R, Weintraub M, Keil LC 1984 Mechanism of suppression of vasopressin and adrenocorticotropic hormone

by clonidine in anesthetized dogs. J Pharmacol Exp Ther 229:1-8
13. Willoughby JO, Jervois PM, Menadue MF, Blessing WW 1987 Noradrenaline, by activation of alpha-1-adrenoceptors in the region of the supraoptic nucleus, causes secretion of vasopressin in the unanesthetized rat. Neuroendocrinology 45:219-226

14. Humphreys MH, Reid IA, Chou LYN 1975 Suppression of antidiuretic hormone secretion by clonidine in the anesthetized dog. Kidney Int 7:405-412

15. Engler D, Pham T, Fullerton MJ, Ooi G, Funder JW, Clarke IJ 1989 Studies of the secretion of corticotropin-releasing factor and arginine vasopressin into the hypophysial-portal circulation of the conscious sheep. Neuroendo-

16. Cheng SWT, North WG 1985 Function of vasopressinergic neurons in rats under conscious and anesthetized conditions. Am J Physiol 248:E155-E161 